\title{
Non-natural protein-protein communication mediated by a DNA- based, antibody-responsive device
}

Simona Ranallo, ${ }^{[a],[b], \#, *}$ Daniela Sorrentino, ${ }^{[a], \#}$ Elisabetta Delibato, ${ }^{[c]}$ Gianfranco Ercolani, ${ }^{[a]}$ Kevin W. Plaxco, ${ }^{[b]}$ and Francesco Ricci ${ }^{[a], *}$

[a] Department of Chemistry, University of Rome, Tor Vergata, Via della Ricerca Scientifica, 00133 Rome, Italy.

[b] Department of Chemistry and Biochemistry, University of California, Santa Barbara, Santa Barbara, CA93106, United States.

[c] Department of Food Safety, Nutrition and Veterinary Public Health, Istituto Superiore di Sanità, Viale Regina Elena 299, Rome, Italy.

*Corresponding author: simona.ranallo@uniroma2.it; francesco.ricci@uniroma2.it

\# These authors contributed equally

\begin{abstract}
We report here the rational design and optimization of an antibody responsive, DNA-based device that enables communication between pairs of otherwise non-interacting proteins. The device is designed to recognize and bind a specific antibody and, in response, undergo a conformational change that leads to the release of a DNA strand, termed the "translator," that regulates the activity of a downstream target protein. As proof of principle, we demonstrate antibody-induced control of the proteins thrombin and Taq DNA polymerase. The resulting strategy is versatile and, in principle, can be easily adapted to control artificial protein-protein communication in artificial regulatory networks.
\end{abstract}




\section{Introduction}

The complex, tightly regulated networks ${ }^{1,2}$ through which DNA, RNA and proteins interact underly the functioning of living systems. ${ }^{3-5}$ One of the aims of synthetic biology is to create artificial pathways in which DNA, RNA and proteins interact with each other via analogously "programmed" reaction patterns to create new tools for sensing, drug-delivery, cell imaging. ${ }^{6-14} \mathrm{~A}$ widely used approach to this end is the rational design of synthetic DNA/protein communication that takes advantage of the many naturally occurring proteins that recognize and bind specific oligonucleotide sequences to, for example, regulate transcription or translation. ${ }^{15-21}$ Such sequence-specific recognition has been employed in synthetic systems to regulate the load/release of molecular cargos from DNA-based devices ${ }^{22}$ the assembly/disassembly of DNA-based structures ${ }^{23}$ and DNA-based reactions. ${ }^{24}$

The chemical versatility of synthetic nucleotides makes it possible to tailor the chemistry by which they can "communicate" with proteins, offering opportunities to expand the abovedescribed regulatory approach to proteins that do not normally bind DNA or RNA. Peptide nucleic acid (PNA)-polypeptide chimeras, for example, have been used to combine the recognition capability of nucleobase sequence and the structural and functional versatility of proteins and peptides into a single molecule. ${ }^{25,26}$ Synthetic oligonucleotides can also be conjugated with other recognition elements responding to a wide range of proteins and biomolecules, further broadening the potential interface between the world of synthetic nucleic acids and proteins. Recently, we and others have, for example, employed antigenconjugated synthetic DNA strands to allow programmable interactions with specific antibodies $^{27-31}$ that control the assembly and disassembly of DNA-based molecular structures. ${ }^{32}$

In the above-described examples, the communication is limited to protein-to-DNA interactions in which a specific protein (e.g., a transcription factor, an enzyme, or an antibody) triggers a functional event in a structure built of DNA. An important step towards the broader applicability of these systems, however, would be the ability to exploit synthetic DNA-based systems as an interface between two normally non-communicating proteins (i.e., protein-DNA-protein communication). A step in this direction was demonstrated by Margulies and coworkers, who employed oligonucleotide-small molecule conjugates to mediate communication between platelet-derived growth factor (PDGF) and glutathione-Stransferase, such that the former controls the catalytic activity of the latter. ${ }^{33}$ 
Motivated by the potential power of DNA devices that modulate protein-protein interactions here we report a class of antibody-responsive, DNA-based synthetic devices that can mediate the regulation of a range of target proteins via two, related mechanisms. In the first, antibody binding to an antigen-conjugated DNA device releases a DNA strand (i.e., translator, green Figure 1, left) that acts as an inhibitor of a downstream target protein (Figure 1, left). In the second, antibody-induced release of the translator strand disrupts the device's inhibition of a downstream protein, activating it (Figure 1, right).
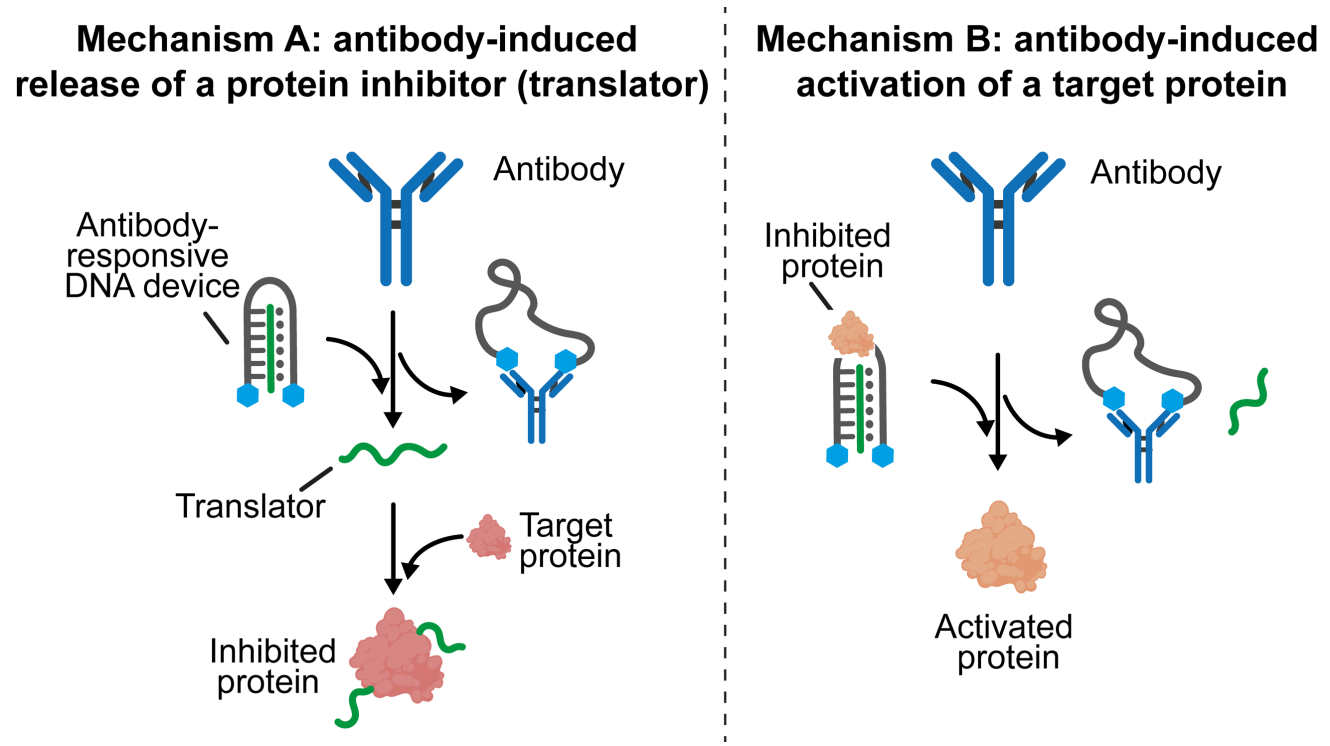

Figure 1. Antibody-protein communication mediated by antibody-responsive DNA devices. Here we explore two mechanisms by which a synthetic, antigen-conjugated DNA strand (antibody-responsive device, grey) can mediate communication between an antibody and a target protein. In the first (Mechanism A), the antibody-responsive device releases a translator strand that inhibits a downstream target protein. (Mechanism B) In the second, antibody-binding-induced loss of the translator abolishes the inhibitory properties of the device, activating the downstream target protein.

\section{Results and discussion}

Our first antibody-responsive device is comprised of a single strand of DNA that forms a hairpin and is modified on each end with an antigen (blue hexagons, Figure 1). ${ }^{34}$ Through the formation of Watson-Crick and Hoogsteen base pairing, this hairpin recognizes and sequesters a second strand of DNA, called the "translator" (green in Figure 1). The bivalent binding of the antibody to the antigen-conjugated strand opens the hairpin, releasing the translator, which is then available to interact with, and thus regulate, a target protein. 
As the first step in the design of our device we characterized and optimized the antibodyinduced release of the translator. Our goal here was to ensure that the translator/device complex is stable enough to limit the release of translator in the absence of the target antibody and yet not so stable that antibody binding cannot easily compete with it. To achieve this, we designed a set of translator variants in which the length of the region complementary to the same antibody-responsive device varied from 8 to 15 bases (Figure S1, S2, S3, see also Supporting Info). To follow the release of the translator we employed a fluorophore-and-quencher labelled duplex that, via a strand displacement reaction, is disrupted upon binding to released translator, increasing the fluorescence signal (Figure 2a). Applying this approach to an antibody-responsive device in which Digoxigenin (Dig) serves as the antigen, we find that translators with 9 or fewer bases complementary to the responsive device do not bind efficiently to it (Figure 2b). Conversely, translators whose complementary regions are greater than 14 bases remain bound even after the addition of the anti-Dig antibody target (Figure 2c). Translators with 10- to 13-base complementary regions, in contrast, are released efficiently only in the presence of anti-Dig antibodies, with a 12-base complementary region achieving the greatest change in free translator concentration upon target binding (Figure 2d).

To characterize our design strategy more quantitatively, we next developed a competitive equilibrium model. In this the responsive device binds either to the translator or to the anti-

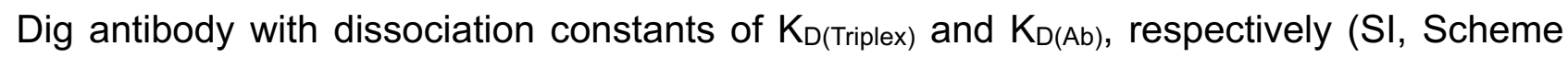
S1). To determine $K_{D \text { (Triplex) }}$ for each translator variant we measured the amount of translator released in the absence of the target antibody (Figure $2 b$ ). As expected, $\mathrm{K}_{\mathrm{D} \text { (Triplex) }}$ is strongly

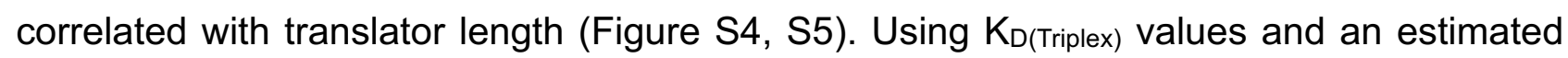
$\mathrm{K}_{\mathrm{D}(\mathrm{Ab})}$ value of $6 \mathrm{nM}$ (see SI for a full discussion of dissociation constants evaluation and the estimated values), we then calculated the expected concentration of free translator as a function of anti-Dig antibody concentration. ${ }^{35}$ Comparison of these values with our experimental measurements (see Figure $2 \mathrm{~d}$ and SI for details) indicate good agreement, confirming the validity of this model. 

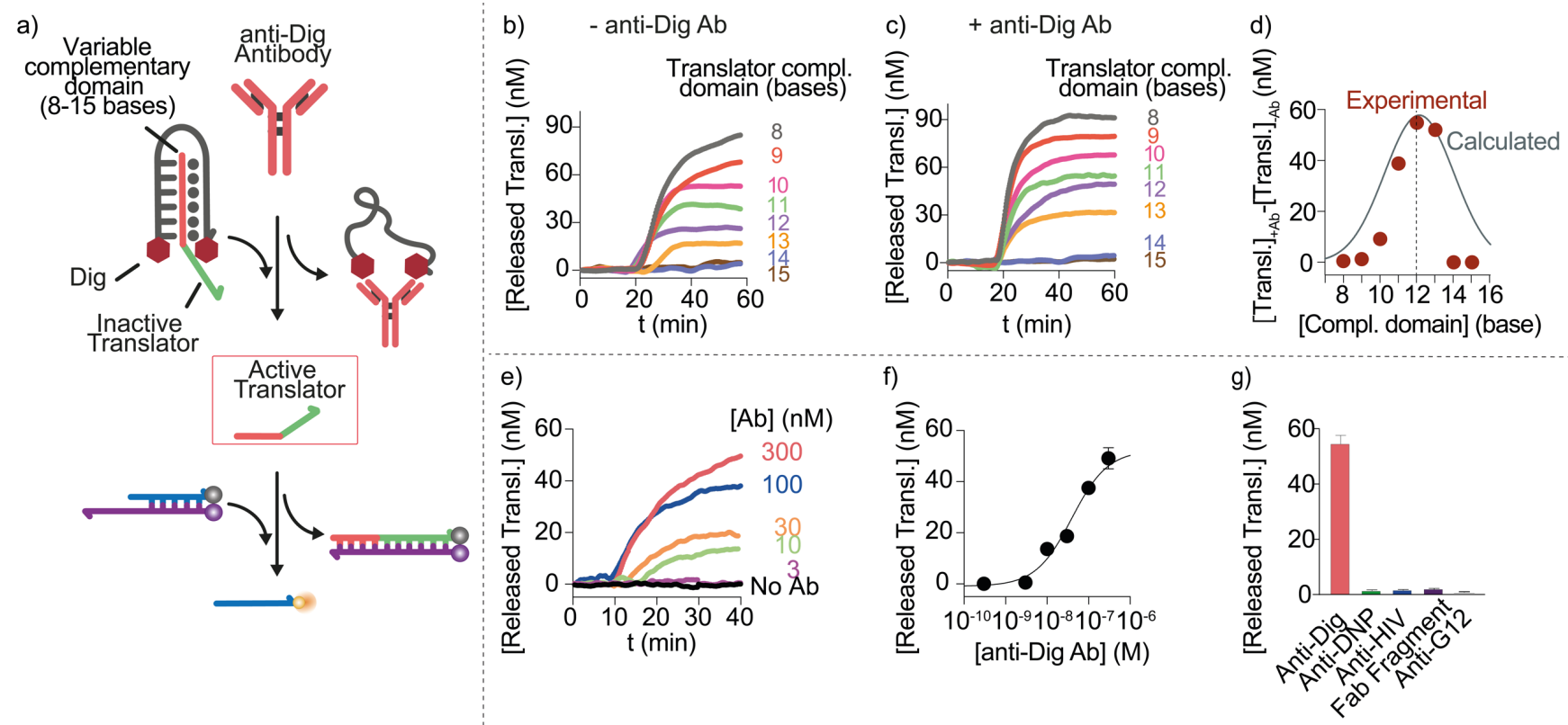

Figure 2. a) Anti-Dig induced release of a translator strand measured through a strand displacement reaction with a dual-labelled duplex strand. b,c) Kinetic traces obtained with the anti-Dig-responsive device for a series of translators differing in the length of the domain complementary to the antibody-responsive device (from 8 to 15 base) in the absence and presence (300 nM) of anti-Dig antibody. d) Plot of experimental (dots) and calculated (grey line, Scheme S1, details in SI) released translator concentration in the presence of anti-Dig antibody. e) Kinetic traces obtained at different concentrations of anti-Dig antibody using the 12-base translator strand. f) Released translator concentration vs. anti-Dig concentration. g) Released translator concentration at saturating concentration (300 nM) of anti-Dig antibodies and different no-targeted antibodies. All the experiments in this and the following figure were performed in a $100 \mu \mathrm{L} 50 \mathrm{mM} \mathrm{Na}_{2} \mathrm{HPO}_{4}, 150 \mathrm{mM} \mathrm{NaCl}, 10 \mathrm{mM} \mathrm{MgCl} 2 \mathrm{pH} 6.5$ buffer solution at $37^{\circ} \mathrm{C}$ employing equimolar concentration of DNA-based anti-Dig responsive device, translator and pre-hybridized fluorophore-and-quencher duplex (100 nM) and the indicated concentration of anti-Dig or no-targeted antibody. In all the sketches, the 3 ' are marked with an arrow.

Translator release is monotonically and quantitatively related to anti-Dig antibody concentration. To demonstrate this, we titrated a device loaded with the optimal, 12-base translator against increasing concentrations of antibody, finding the expected Langmuir isotherm relationship between antibody concentration and translator release (Figure 2e-f). In contrast, no release is observed in the presence of other, non-targeted antibodies (Figure $2 \mathrm{~g}, \mathrm{S6}$ ). By replacing the antibody-binding antigens our antibody-responsive devices can be adapted to other antibodies. We demonstrate this using dinitrophenol (DNP) as the antigen and anti-DNP and input antibody. With this we achieved antibody responsiveness and specificity comparable to those observed for the anti-Dig responsive device (Figure S7-S11). Of potential future relevance, the two antibody-responsive devices can be orthogonally 
controlled: by mixing the two in the same solution and challenging them with various combinations of their target antibodies we find that each responds only to its specific target antibody (Figure S12, 13, 14).

The antibody-responsive device supports efficient communication between normally noninteracting proteins. As our first example we designed a device that achieves antibodyinduced downstream regulation of thrombin, a key protein in coagulant functions that leads to cleavage of fibrinogen into fibrin monomers (Figure 3a). For this we employed a 15-base DNA aptamer as our translator that binds to thrombin and inhibits its proteolytic activity. ${ }^{36-38}$ We re-engineered this to incorporate a domain that forms a triplex with our anti-Dig-antibody responsive device (grey in Figure 3a). In the absence of anti-Dig antibodies (black curve Figure $3 b)$ or at high concentrations (300 nM) of a control antibody (grey curve Figure $3 b$ ) this device does not measurably inhibit thrombin activity. In the presence of anti-Dig antibodies, in contrast, we observe a significantly longer coagulation lag-time (time before significant signal rise), indicating that the released translator is inhibiting thrombin activity (Figure 3b). As expected, the inhibition increases monotonically with increasing concentration of the antibody (Figure 3c, S15).
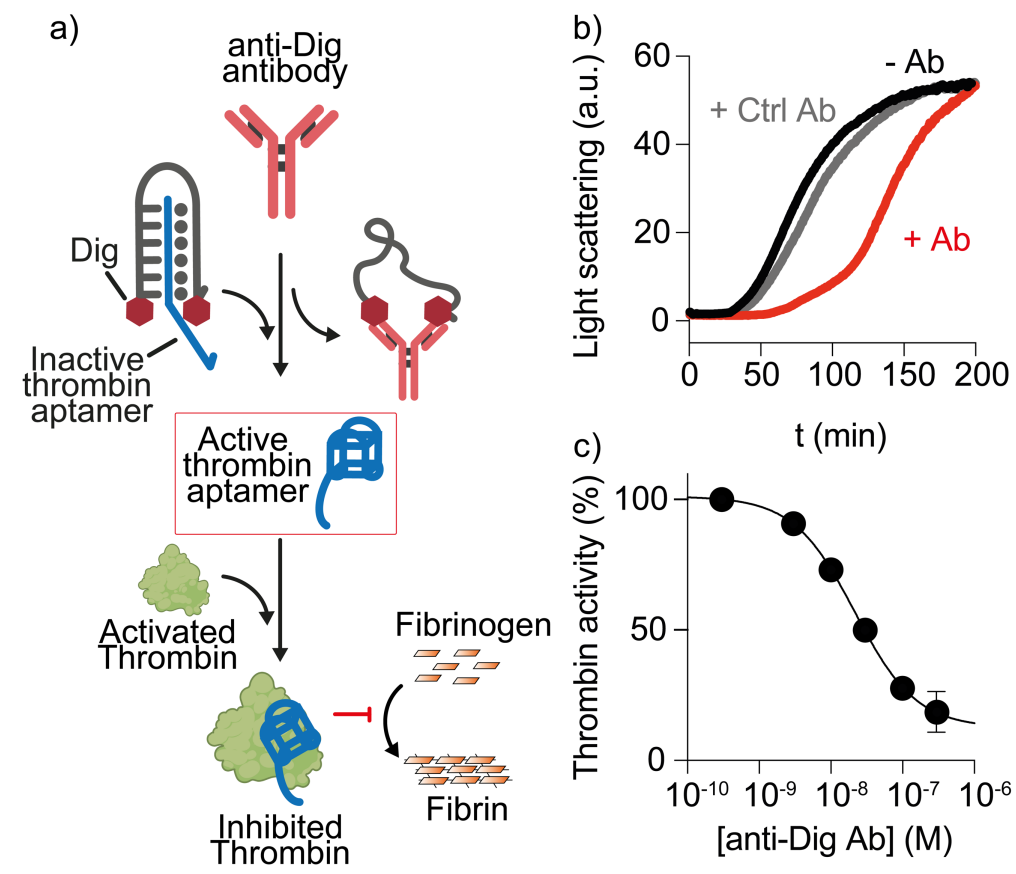

Figure 3. a) Here we have used antibody-protein communication to regulate the proteolytic activity of thrombin. b) Thrombin-mediated fibrin formation followed by light scattering increase after addition of fibrinogen $(1 \mathrm{mg} / \mathrm{mL})$ to a solution containing equimolar concentration of DNA responsive device/Thrombin Translator (100 nM) and thrombin (1 nM) in the absence and in the presence of anti-Dig antibody or a control antibody (300 nM). c) Thrombin activity varies as a function of antibody concentration. Experimental conditions (buffer, $\mathrm{pH}$, temperature) as indicated in Figure 2. 
Our responsive devices are generalizable to the control of other proteins. To illustrate this we engineered a second device architecture (Figure 1, right and Figure 4a) in which the antigen-modified strand of the device itself acts as the translator. To do this we employed a triplex-forming DNA aptamer that binds and inhibits Taq DNA polymerase as the triplex. ${ }^{39}$ In this new architecture we conjugated copies of the antigen digoxigenin directly on to the two ends of the triplex forming aptamer and employed a 12-base DNA cognate strand that induces efficient triplex formation, and thus aptamer activity. In the absence of anti-Dig antibodies (black curve, Figure 4b) or at saturating concentration of a non-targeted antibody (grey curve, Figure 4b) the system efficiently inhibits Taq DNA polymerase activity. Upon antibody binding, however, the triplex is disrupted, resulting in the recovery of enzymatic activity (Figure 4b, red curve, Figure S16). Quantitative regulation of polymerase activity is once again easily achieved by varying the concentration of the target antibody (Figure 4c, S17).

a)

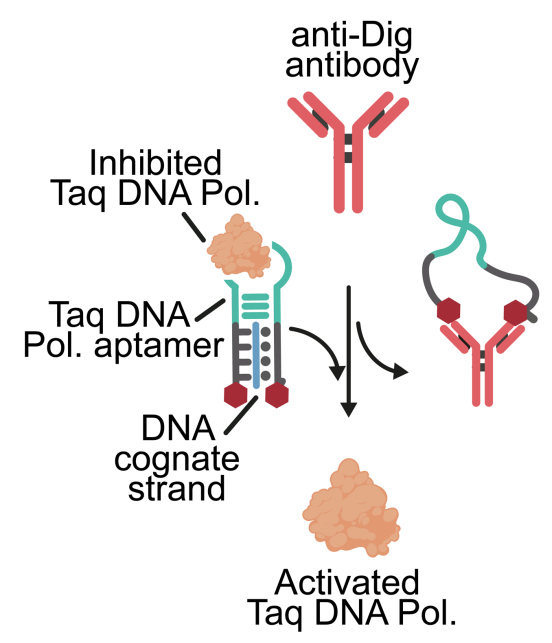

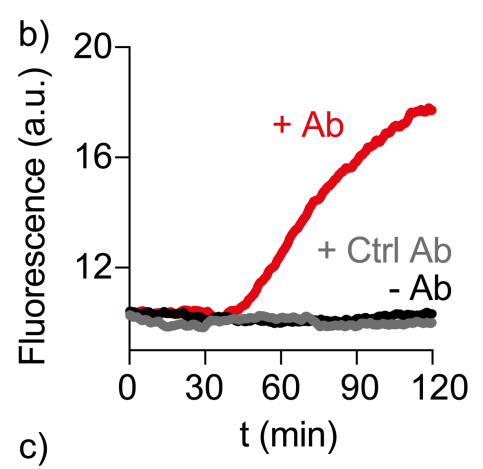

c)

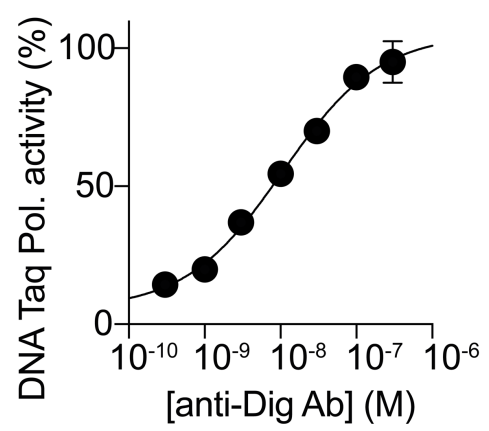

Figure 4. a) Antibody-protein communication to control Taq DNA polymerase activity. b) Taq DNA polymerase activation followed through kinetic traces in presence of anti-Dig antibodies (300 nM). No activation is observed in absence of anti-Dig antibodies and at high concentrations (300 nM) of a control antibody (grey curve). c) Anti-Dig antibodies concentration-dependent regulation of Taq polymerase activity. For experimental conditions see SI. 


\section{Conclusion}

Information processing in living cells results from the communication between DNA, RNA and proteins. ${ }^{1-3}$ Drawing inspiration from these naturally occurring molecular communication systems, we have exploited the designability of synthetic DNA to create molecular devices that allow the regulation of one protein by another. Specifically, we developed antibodyresponsive nanodevices able to release DNA translators in presence of specific antibodies, thus regulating the activity of downstream target proteins. This strategy is versatile and, in principle, can be easily adapted to modulate artificial protein-DNA and protein-protein communication mediated by DNA-based devices. Re-engineering programmed networks with well-defined behaviors represents the main aim of synthetic biology thus allowing advanced functionality in various biological applications, such as molecular computing, biochemical sensing and nanomedicine. ${ }^{6,12}$ 


\section{Acknowledgment}

This work received funding from the European Union's Horizon 2020 research and innovation program under the Marie Skłodowska-Curie grant agreement n. 843179 ("DNANANO-AB", S.R.). The work was also supported by Associazione Italiana per la Ricerca sul Cancro, AIRC (project n. 21965 F.R.) and by the European Research Council, ERC (Consolidator Grant project n. 819160, F.R.).

\section{References}

(1) Liu, X.; Maiorino, E.; Halu, A.; Glass, K.; Prasad, R. B.; Loscalzo, J.; Gao, J.; Sharma, A. Robustness and lethality in multilayer biological molecular networks. Nat. Commun. 2020, 11, 6043.

(2) Park, S.; Prévost, K.; Heideman, E.M.; Carrier, M.-C.; Azam, M. S.; Reyer, M. A.; Liu, W.; Massé, E.; Fei, J. Dynamic interactions between the RNA chaperone Hfq, small regulatory RNAs and mRNAs in live bacterial cells. eLife 2021, 10, 1-45.

(3) Duan, N.; Arroyo, M.; Deng, W.; Cardoso, M.C.; Leonhardt H. Visualization and characterization of RNA-protein interactions in living cells. Nucleic Acids Res. 2021, 49, e107.

(4) Moore, M.J. From birth to death: the complex lives of eukaryotic mRNAs. Science 2005, 309, 1514-1518.

(5) Keene, J.D. RNA regulons: coordination of post transcriptional events. Nat. Rev. Genet. 2007, 8, 533-543.

(6) Yue, L.; Wulf, V.; Wang, S.; Willner, I. Evolution of Nucleic-Acid-Based Constitutional Dynamic Networks Revealing Adaptive and Emergent Functions. Angew. Chem. Int. Ed. 2019, 58, 12238-12245.

(7) Chen, Y.-J.; Groves, B.; Muscat, R. A.; Seelig, G. DNA nanotechnology from the test tube to the cell. Nat. Nanotechnol. 2015, 10, 748-760.

(8) Xiao, M; Lai, W.; Man, T.; Chang, B.; Li, L.; Chandrasekaran, A. R.; Pei. H. Rationally Engineered Nucleic Acid Architectures for Biosensing Applications. Chem. Rev. 2019, 119, 11631-11717.

(9) Watson, E. E.; Angerani, S.; Sabale, P. M.; Winssinger, N. Biosupramolecular Systems: Integrating Cues into Responses. J. Am. Chem. Soc. 2021, 143, 4467-4482. 
(10) Mashima, T.; Rosier, B.J.H.M.; Oohora, K.; de Greef, T.F.A.; Hayashi, T.; Brunsveld, L. Dynamic Protease Activation on a Multimeric Synthetic Protein Scaffold via Adaptable DNA-Based Recruitment Domains. Angew. Chem. Int. Ed. 2021, 60, 1126211266.

(11) Peng, R.; Xu, L.; Wang, H.; Lyu, Y.; Wang, D.; Bi, C.; Cui, C.; Fan, C.; Liu, Q.; Zhang, X.; Tan, W. DNA-based artificial molecular signaling system that mimics basic elements of reception and response. Nat. Commun. 2020, 11, 978.

(12) Yue, L.; Wang, S.; Zhou, Z.; Willner, I. Nucleic Acid Based Constitutional Dynamic Networks: From Basic Principles to Applications. J. Am. Chem. Soc. 2020, 142, 2157721594.

(13) Joesaar, A.; Yang, S.; Bögels, B.; van der Linden, A.; Pieters, P.; Kumar, B.V.V.S.P.; Dalchau, N.; Phillips, A.; Mann, S.; de Greef, T.F.A. DNA-based communication in populations of synthetic protocells. Nat. Nanotechnol. 2019, 14, 369-378.

(14) Engelen, W.; Janssen, B.M.G.; Merkx, M. DNA-based control of protein activity. Chem. Commun. 2016, 52, 3598-3610.

(15) Badis, G.; Berger, M. F.; Philippakis, A. A.; Talukder, S.; Gehrke, A. R.; Jaeger, S. A.; Chan, E. T.; Metzler, G.; Vedenko, A.; Chen, X.; Kuznetsov, H.; Wang, C.-F.; Coburn, D.; Newburger, D. E.; Morris, Q.; Hughes, T. R.; Bulyk, M.L. Diversity and complexity in DNA recognition by transcription factors. Science 2009, 324, 1720-1723.

(16) Heyduk, T.; Heyduk, E. Molecular beacons for detecting DNA binding proteins. Nat. Biotechnol. 2002, 20, 171-176.

(17) Bertucci, A.; Guo, J.; Oppmann, N.; Glab, A.; Ricci, F.; Caruso, F.; Cavalieri, F. Probing transcription factor binding activity and downstream gene silencing in living cells with a DNA nanoswitch. Nanoscale 2018, 10, 2034-2044.

(18) Vallée-Bélisle, A.; Bonham, A. J.; Reich, N. O.; Ricci, F.; Plaxco, K. W. Transcription factor beacons for the quantitative detection of DNA binding activity. J. Am. Chem. Soc. 2011, 133, 13836-13839.

(19) Bonham, A. J.; Hsieh, K.; Ferguson, B. S.; Vallée-Bélisle, A.; Ricci, F.; Soh, H. T.; Plaxco, K. W. Quantification of transcription factor binding in cell extracts using an electrochemical, structure-switching biosensor. J. Am. Chem. Soc. 2012, 134, 33463348. 
(20) Etheve, L.; Martin, J.; Lavery, R. Protein-DNA interfaces: a molecular dynamics analysis of time-dependent recognition processes for three transcription factors. Nucleic Acids Res. 2016, 44, 9990-10002.

(21) Lambert, S.A.; Jolma, A.; Campitelli, L.F.; Das, P.K.; Yin, Y.; Albu, M.; Chen, X.; Taipale, J.; Hughes, T.R.; Weirauch, M.T. The Human Transcription Factors. Cell 2018, $172,650-665$.

(22) Rossetti, M.; Ranallo, S.; Idili, A.; Palleschi, G.; Porchetta, A.; Ricci, F. Allosteric DNA nanoswitches for controlled release of a molecular cargo triggered by biological inputs. Chem. Sci. 2017, 8, 914-920.

(23) Crawford, R.; Erben, C.M.; Periz, J.; Hall, L.M.; Brown, T.; Turberfield, A.J.; Kapanidis, A.N. Non-covalent single transcription factor encapsulation inside a DNA cage. Angew. Chem. Int. Ed. 2013, 52, 2284-2288.

(24) Bertucci, A.; Porchetta, A.; Del Grosso, E.; Patiño, T.; Idili, A.; Ricci, F. ProteinControlled Actuation of Dynamic Nucleic Acid Networks by Using Synthetic DNA Translators. Angew. Chem. Int. Ed. 2020, 59, 20577-20581.

(25) Swenson, C. S.; Velusamy, A.; Argueta-Gonzalez, H. S.; Heemstra, J. M. Bilingual Peptide Nucleic Acids: Encoding the Languages of Nucleic Acids and Proteins in a Single Self-Assembling Biopolymer. J. Am. Chem. Soc. 2019, 141, 19038-19047

(26) Gavins, G. C.; Gröger, K.; Bartoschek, M. D.; Wolf, P.; Beck-Sickinger, A. G.; Bultmann, S.; Seitz, O. Live cell PNA labelling enables erasable fluorescence imaging of membrane proteins. Nat. Chem. 2021, 13, 15-23.

(27) Baranda, L. P.; Mahdifar, M.; Ercolani, G.; Watson, J.; Brown, Jr. T.; Ricci, F. Using antibodies to control DNA-templated chemical reactions. Nat. Commun. 2020, 11, 6242.

(28) Janssen, B.M.G.; Lempens, E.H.M.; Olijve, L.L.C.; Voets, I.K.; van Dongen, J.L.J.; de Greef, T.F.A.; Merkx, M. Reversible blocking of antibodies using bivalent peptide-DNA conjugates allows protease-activatable targeting. Chem. Sci. 2013, 4, 1442-1450.

(29) Janssen, B.M.G.; van Rosmalen, M.; van Beek, L.; Merkx, M. Antibody activation using DNA-based logic gates. Angew. Chem. Int. Ed. 2015, 54, 2530-2533.

(30) Engelen, W.; Meijer, L.H.H.; Somers, B.; De Greef, T.F.A.; Merkx, M. Antibodycontrolled actuation of DNA-based molecular circuits. Nat. Commun. 2017, 8, 14473 
(31) Rosier, B.J.H.M.; Cremers, G.A.O.; Engelen, W.; Merkx, M.; Brunsveld, L.; De Greef, T.F.A. Incorporation of native antibodies and Fc-fusion proteins on DNA nanostructures via a modular conjugation strategy (2017) ChemComm. 2017, 53, 7393-7396.

(32) Ranallo, S.; Sorrentino, D.; Ricci, F. Orthogonal regulation of DNA nanostructure selfassembly and disassembly using antibodies. Nat. Commun. 2019, 10, 5509.

(33) Peri-Naor, R.; Ilani, T.; Motiei, L.; Margulies, D. Protein-Protein Communication and Enzyme Activation Mediated by a Synthetic Chemical Transducer. J. Am. Chem. Soc. 2015, 137, 9507-9510.

(34) Ranallo, S.; Prévost-Tremblay, C.; Idili, A.; Vallée-Bélisle, A.; Ricci F. Antibodypowered nucleic acid release using a DNA-based nanomachine. Nat. Commun. 2017, 8, 15150.

(35) Hoops, S.; Sahle, S.; Gauges, R.; Lee, C.; Pahle, J.; Simus, N.; Singhal, M.; Xu, L.; Mendes, P.; Kummer, U. COPASI: a COmplex PAthway SImulator. Bioinformatics 2006, 22, 3067-74.

(36) Bock, L. C.; Griffin, L. C.; Latham, J. A.; Vermaas, E. H.; Toole, J. J. Selection of single-stranded DNA molecules that bind and inhibit human thrombin. Nature 1992 , 355, 564-566.

(37) Yoshida, W.; Sode, K.; Ikebukuro, K. Homogeneous DNA Sensing Using EnzymeInhibiting DNA Aptamers. Biochem. Biophys. Res. Commun. 2006, 348, 245-252.

(38) Shiang, Y.C.; Huang, C.C.; Wang, T.H.; Chien, C.W.; Chang, H.T. AptamerConjugated Nanoparticles Efficiently Control the Activity of Thrombin. Adv. Funct. Mater. 2010, 20, 3175- 3182.

(39) Hu, Y.; Wang, Z.; Chen, Z.; Pan, L. Switching the activity of Taq polymerase using clamp-like triplex aptamer structure. Nucleic Acids Res. 2020, 48, 8591-8600. 